\title{
On the positive linear set valued maps
}

Anar Huseyin and Nesir Huseyin 


\title{
ON THE POSITIVE LINEAR SET VALUED MAPS
}

\author{
ANAR HUSEYIN AND NESIR HUSEYIN
}

Received October 21, 2011

\begin{abstract}
In this paper positive linear set valued maps defined on the cone are studied. The representation theorem for positive linear set valued maps is given and Lipschitz continuity of these maps is proved. The estimations of upper and lower norms of the positive linear set valued maps are obtained.
\end{abstract}

2000 Mathematics Subject Classification: 54C60; 54C65

Keywords: positive linear set valued map, representation of set valued maps

\section{INTRODUCTION}

It is known that the set valued analogues of continuous linear operators are set valued maps the graph of which are cones or vector subspaces. These kinds of set valued maps are called processes or linear processes respectively (see, e.g. [10,11]). They provide a good framework for investigation of general homogenous systems which arise in economics, mechanics and functional analysis (see, e.g. [2, 3, 7, 12]). Differentials of the set valued maps provide examples of closed convex processes (see, e.g. [2, 4]). Various properties of the convex processes are investigated in [1$3,5,6,8-12]$. Regularity and continuity of the convex processes are discussed in $[2,3,5,6,8,9,12]$. In [1] controllability property of the differential inclusion is studied where the right hand side of the given differential inclusion is a convex process. In this paper positive linear set valued maps are defined which satisfy additivity and positive homogeneity conditions. The paper is organized as follows:

In Section 2 the representation theorem for a positive linear set valued map is given (Theorem 1). In Section 3 the Lipschitz continuity of the positive linear set valued maps is proved (Theorem 2). In Section 4 the evaluations of upper and lower norms of the positive linear set valued maps are obtained (Theorem 3 ).

Let $\mathbb{R}^{n}$ be the $n$-dimensional Euclidean space, $\left(X,\|\cdot\|_{X}\right)$ and $\left(Y,\|\cdot\|_{Y}\right)$ be normed spaces, and $a_{i} \in X, i=1,2, \ldots, n$, are nonzero linearly independent vectors. Denote

$$
\mathbb{R}_{+}^{n}=\left\{\xi=\left(\xi_{1}, \xi_{2}, \ldots, \xi_{n}\right) \in \mathbb{R}^{n}: \xi_{i} \geq 0, i=1,2, \ldots, n\right\},
$$




$$
\begin{gathered}
K=\left\{x=\sum_{i=1}^{n} \xi_{i} a_{i}:\left(\xi_{1}, \xi_{2}, \ldots, \xi_{n}\right) \in \mathbb{R}_{+}^{n}\right\} \\
E^{n}=\left\{x=\sum_{i=1}^{n} \xi_{i} a_{i}:\left(\xi_{1}, \xi_{2}, \ldots, \xi_{n}\right) \in \mathbb{R}^{n}\right\} \\
\alpha=\left(\sum_{i=1}^{n}\left\|a_{i}\right\|_{X}^{2}\right)^{\frac{1}{2}} .
\end{gathered}
$$

It is obvious that $K \subset X$ is a cone and every $x \in K$ has a unique representation in the form $x=\sum_{i=1}^{n} \xi_{i} a_{i}$, where $\xi=\left(\xi_{1}, \xi_{2}, \ldots, \xi_{n}\right) \in \mathbb{R}_{+}^{n}$. Similarly, every $x \in E^{n}$ also has a unique representation in the form $x=\sum_{i=1}^{n} \xi_{i} a_{i}$, where $\xi=\left(\xi_{1}, \xi_{2}, \ldots, \xi_{n}\right) \in \mathbb{R}^{n}$. For given $x \in E^{n}$ we will denote $\mathbf{x}_{n}=\left(\xi_{1}, \xi_{2}, \ldots, \xi_{n}\right) \in \mathbb{R}^{n}$ and $\left\|\mathbf{x}_{n}\right\|=\left(\sum_{i=1}^{n} \xi_{i}^{2}\right)^{\frac{1}{2}}$. For each $\xi=\left(\xi_{1}, \xi_{2}, \ldots, \xi_{n}\right) \in \mathbb{R}^{n}$ we define a function $f(\cdot): \mathbb{R}^{n} \rightarrow[0, \infty)$, setting

$$
f(\xi)=f\left(\xi_{1}, \xi_{2}, \ldots, \xi_{n}\right)=\left\|\sum_{i=1}^{n} \xi_{i} a_{i}\right\|_{X}
$$

Let

$$
\begin{gathered}
S^{n}=\left\{\xi=\left(\xi_{1}, \xi_{2}, \ldots, \xi_{n}\right) \in \mathbb{R}^{n}:\|\xi\|=1\right\}, \\
S_{+}^{n}=\left\{\xi=\left(\xi_{1}, \xi_{2}, \ldots, \xi_{n}\right) \in \mathbb{R}_{+}^{n}:\|\xi\|=1\right\} .
\end{gathered}
$$

It is clear that $S_{+}^{n} \subset \mathbb{R}^{n}, S^{n} \subset \mathbb{R}^{n}$ are compact sets, $S_{+}^{n} \subset S^{n}, f(\cdot): \mathbb{R}^{n} \rightarrow[0, \infty)$ is a continuous function and

$$
f(\xi)=\left\|\sum_{i=1}^{n} \xi_{i} a_{i}\right\|_{X}>0 \text { for every } \xi=\left(\xi_{1}, \xi_{2}, \ldots, \xi_{n}\right) \in S^{n} .
$$

Hence there exist $\gamma>0$ and $\beta>0$ such that

$$
\begin{aligned}
& \min \left\{f(\xi): \xi \in S^{n}\right\}=\gamma, \\
& \min \left\{f(\xi): \xi \in S_{+}^{n}\right\}=\beta
\end{aligned}
$$

It is obvious that $\beta \geq \gamma>0$.

Proposition 1. The inequality

$$
\gamma\left\|\mathbf{x}_{n}\right\| \leq\|x\|_{X} \leq \alpha\left\|\mathbf{x}_{n}\right\|
$$

is satisfied for every $x \in E^{n}$, and the inequality

$$
\beta\left\|\mathbf{x}_{n}\right\| \leq\|x\|_{X} \leq \alpha\left\|\mathbf{x}_{n}\right\|
$$


holds for every $x \in K$, where $\alpha>0, \gamma>0$ and $\beta>0$ are defined by (1.3), (1.5) and (1.6) respectively.

Proof. Let $x \in E^{n}$. Then $x=\sum_{i=1}^{n} \xi_{i} a_{i}$, where $\xi=\left(\xi_{1}, \xi_{2}, \ldots, \xi_{n}\right) \in \mathbb{R}^{n}$, and hence $\mathbf{x}_{n}=\left(\xi_{1}, \xi_{2}, \ldots, \xi_{n}\right) \in \mathbb{R}^{n}$. Thus, using Cauchy-Schwarz inequality, we obtain from (1.3) that

$$
\|x\|_{X}=\left\|\sum_{i=1}^{n} \xi_{i} a_{i}\right\|_{X} \leq \sum_{i=1}^{n}\left|\xi_{i}\right|\left\|a_{i}\right\|_{X} \leq\left(\sum_{i=1}^{n} \xi_{i}^{2}\right)^{\frac{1}{2}} \cdot\left(\sum_{i=1}^{n}\left\|a_{i}\right\|_{X}^{2}\right)^{\frac{1}{2}}=\alpha\left\|\mathbf{x}_{n}\right\| .
$$

Now, let again $x=\sum_{i=1}^{n} \xi_{i} a_{i} \in E^{n}$ be an arbitrarily chosen vector where $x \neq 0$. Then $\mathbf{x}_{n}=\left(\xi_{1}, \xi_{2}, \ldots, \xi_{n}\right) \in \mathbb{R}^{n}, \mathbf{x}_{n} \neq 0$ and

$$
\|x\|_{X}=\left\|\sum_{i=1}^{n} \xi_{i} a_{i}\right\|_{X}=\left\|\mathbf{x}_{n}\right\| \cdot\left\|\sum_{i=1}^{n} \frac{\xi_{i}}{\left\|\mathbf{x}_{n}\right\|} \cdot a_{i}\right\|_{X} .
$$

Since $\left(\frac{\xi_{1}}{\left\|\mathbf{x}_{n}\right\|}, \frac{\xi_{2}}{\left\|\mathbf{x}_{n}\right\|}, \ldots, \frac{\xi_{n}}{\left\|\mathbf{x}_{n}\right\|}\right) \in S^{n}$, we have from (1.4) and (1.5) that

$$
\left\|\sum_{i=1}^{n} \frac{\xi_{i}}{\left\|\mathbf{x}_{n}\right\|} \cdot a_{i}\right\|_{X} \geq \gamma
$$

(1.10) and (1.11) imply

$$
\|x\|_{X} \geq \gamma\left\|\mathbf{x}_{n}\right\| .
$$

If $x=0$, then inequality (1.12) is satisfied trivially. From (1.9) and (1.12) we obtain the proof of the inequality (1.7).

Since $K \subset E^{n}$, (1.7) gives

$$
\|x\|_{X} \leq \alpha\left\|\mathbf{x}_{n}\right\|
$$

for every $x \in K$, where $\alpha>0$ is defined by (1.3).

Let, now, $x=\sum_{i=1}^{n} \xi_{i} a_{i} \in K$ be an arbitrarily chosen vector such that $x \neq 0$. Then $\mathbf{x}_{n}=\left(\xi_{1}, \xi_{2}, \ldots, \xi_{n}\right) \in \mathbb{R}_{+}^{n}, \mathbf{x}_{n} \neq 0$ and

$$
\|x\|_{X}=\left\|\sum_{i=1}^{n} \xi_{i} a_{i}\right\|_{X}=\left\|\mathbf{x}_{n}\right\| \cdot\left\|\sum_{i=1}^{n} \frac{\xi_{i}}{\left\|\mathbf{x}_{n}\right\|} \cdot a_{i}\right\|_{X} .
$$


Since $\left(\frac{\xi_{1}}{\left\|\mathbf{x}_{n}\right\|}, \frac{\xi_{2}}{\left\|\mathbf{x}_{n}\right\|}, \ldots, \frac{\xi_{n}}{\left\|\mathbf{x}_{n}\right\|}\right) \in S_{+}^{n}$, we have from (1.4) and (1.6) that

$$
\left\|\sum_{i=1}^{n} \frac{\xi_{i}}{\left\|\mathbf{x}_{n}\right\|} \cdot a_{i}\right\|_{X} \geq \beta .
$$

(1.14) and (1.15) imply

$$
\|x\|_{X} \geq \beta\left\|\mathbf{x}_{n}\right\| .
$$

The inequality (1.16) is also verified, if $x=0$. (1.13) and (1.16) yield the proof of the inequality (1.8).

Proposition 2. The cone $K \subset X$ defined by relation (1.1) is a pointed cone, i.e. $K$ is closed, convex, and $K \bigcap(-K)=\left\{0_{X}\right\}$.

Proof. Convexity of the cone $K$ is obvious. Let us prove closedness. Let $x^{(m)}=$ $\sum_{i=1}^{n} \xi_{i}^{(m)} a_{i} \in K$ for every $m=1,2, \ldots$ and $x^{(m)} \rightarrow x^{*}$ as $m \rightarrow \infty$. By virtue of Proposition 1 we have

$$
\left\|\mathbf{x}_{n}^{(m)}\right\| \leq \frac{1}{\beta}\left\|x^{(m)}\right\|_{X}
$$

for every $m=1,2, \ldots$ where $\mathbf{x}_{n}^{(m)}=\left(\xi_{1}^{(m)}, \xi_{2}^{(m)}, \ldots, \xi_{n}^{(m)}\right)$.

Since $x^{(m)} \rightarrow x^{*}$ as $m \rightarrow \infty$, then it follows from (1.17) that there exists an $r>0$ such that

$$
\left\|\mathbf{x}_{n}^{(m)}\right\| \leq r
$$

for every $m=1,2, \ldots$, and hence

$$
\sum_{i=1}^{n}\left(\xi_{i}^{(m)}\right)^{2} \leq r^{2}
$$

for every $m=1,2, \ldots$ (1.18) implies that the sequences $\left\{\xi_{i}^{(m)}\right\}_{m=1}^{\infty}$ are bounded for every $i=1,2, \ldots, n$. Hence there exists a subsequence $\left\{\xi_{i}^{\left(m_{j}\right)}\right\}_{j=1}^{\infty}$ such that $\xi_{i}^{\left(m_{j}\right)} \rightarrow \xi_{i}^{*}$ as $j \rightarrow \infty$ for every $i=1,2, \ldots, n$. It is obvious that $\xi_{i}^{*} \geq 0$ for every $i=1,2, \ldots, n$.

Denote $y_{*}=\sum_{i=1}^{n} \xi_{i}^{*} a_{i}$. Then $y_{*} \in K$ and $x^{\left(m_{j}\right)} \rightarrow y^{*}$ as $j \rightarrow \infty$, where $x^{\left(m_{j}\right)}=$ $\sum_{i=1}^{n} \xi_{i}^{\left(m_{j}\right)} a_{i}$. Since $x^{(m)} \rightarrow x^{*}$ as $m \rightarrow \infty$, we get $x_{*}=y_{*} \in K$. 
Let us prove the validity of the relation

$$
K \bigcap(-K)=\left\{0_{X}\right\} .
$$

Let us assume contrary, i.e. let there exists $x_{*} \in K$ such that $x_{*} \neq 0$ and $-x_{*} \in K$. Then there exist unique $\xi=\left(\xi_{1}, \xi_{2}, \ldots, \xi_{n}\right) \in \mathbb{R}_{+}^{n}$ and $\eta=\left(\eta_{1}, \eta_{2}, \ldots, \eta_{n}\right) \in \mathbb{R}_{+}^{n}$ such that

$$
x_{*}=\sum_{i=1}^{n} \xi_{i} a_{i},-x_{*}=\sum_{i=1}^{n} \eta_{i} a_{i} .
$$

(1.19) yields

$$
\sum_{i=1}^{n}\left(\xi_{i}+\eta_{i}\right) a_{i}=0
$$

Since the vectors $a_{1}, a_{2}, \ldots, a_{n}$ are linearly independent, $\xi=\left(\xi_{1}, \xi_{2}, \ldots, \xi_{n}\right) \in \mathbb{R}_{+}^{n}$ and $\eta=\left(\eta_{1}, \eta_{2}, \ldots, \eta_{n}\right) \in \mathbb{R}_{+}^{n},(1.20)$ implies that $\xi_{i}=0, \eta_{i}=0$ for every $i=1,2, \ldots, n$, and hence $x_{*}=0$. This contradicts with $x_{*} \neq 0$.

The family of all non-empty, compact, and convex subsets of $Y$ is denoted by $P_{k c}(Y)$. The Hausdorff distance between the sets $U \subset Y$ and $V \subset Y$ is denoted by $h_{Y}(U, V)$ and defined as

$$
h_{Y}(U, V)=\max \left\{\sup _{u \in U} d_{Y}(u, V), \sup _{v \in V} d_{Y}(v, U)\right\},
$$

where $d_{Y}(u, V)=\inf \left\{\|u-v\|_{Y}: v \in V\right\}$ (see, e.g., [2]). It is known that $\left(P_{k c}(Y), h_{Y}(\cdot, \cdot)\right)$ is a metric space.

Now let us formulate some properties of convex sets. For $U \in P_{k c}(Y), V \in$ $P_{k c}(Y)$, and $\lambda \in \mathbb{R}^{1}$ we denote

$$
U+V=\{u+v: u \in U, v \in V\}, \lambda U=\{\lambda u: u \in U\} .
$$

It is obvious that if $U \in P_{k c}(Y), V \in P_{k c}(Y), \lambda \in \mathbb{R}^{1}$, then $\lambda(U+V)=\lambda U+\lambda V$. If $U \in P_{k c}(Y), \lambda \in \mathbb{R}_{+}^{1}, \mu \in \mathbb{R}_{+}^{1}$, then $(\lambda+\mu) U=\lambda U+\mu U$.

For a given $U \in P_{k c}(Y)$ we denote

$$
\|U\|=\max \left\{\|u\|_{Y}: u \in U\right\} .
$$

It is not a difficult matter to verify that $\|U\|=0$ iff $U=\left\{0_{Y}\right\},\|U+V\| \leq\|U\|+$ $\|V\|$ for every $U \in P_{k c}(Y)$ and $V \in P_{k c}(Y),\|\lambda U\|=|\lambda|\|U\|$ for every $\lambda \in \mathbb{R}^{1}$ and $U \in P_{k c}(Y)$. Let

$$
\left(P_{k c}(Y)\right)^{n}=\underbrace{P_{k c}(Y) \times P_{k c}(Y) \times \ldots \times P_{k c}(Y)}_{n \text { times }} .
$$

For $\lambda \in \mathbb{R}_{+}^{1}, \xi=\left(\xi_{1}, \xi_{2}, \ldots, \xi_{n}\right) \in \mathbb{R}_{+}^{n}, \quad U=\left(U_{1}, U_{2}, \ldots, U_{n}\right) \in\left(P_{k c}(Y)\right)^{n}, \mathcal{V}=$ $\left(V_{1}, V_{2}, \ldots, V_{n}\right) \in\left(P_{k c}(Y)\right)^{n}$ we denote

$$
u+\mathcal{V}=\left(U_{1}+V_{1}, U_{2}+V_{2}, \ldots, U_{n}+V_{n}\right),
$$




$$
\begin{aligned}
\lambda u & =\left(\lambda U_{1}, \lambda U_{2}, \ldots, \lambda U_{n}\right), \\
\|u\|_{C} & =\left(\sum_{i=1}^{n}\left\|U_{i}\right\|^{2}\right)^{\frac{1}{2}}, \\
<\xi, u> & =\sum_{i=1}^{n} \xi_{i} U_{i} .
\end{aligned}
$$

Proposition 3. Let $U=\left(U_{1}, U_{2}, \ldots, U_{n}\right) \in\left(P_{k c}(Y)\right)^{n}, \mathcal{V}=\left(V_{1}, V_{2}, \ldots, V_{n}\right) \in$ $\left(P_{k c}(Y)\right)^{n}, \lambda \in \mathbb{R}_{+}^{1}, \xi=\left(\xi_{1}, \xi_{2}, \ldots, \xi_{n}\right) \in \mathbb{R}_{+}^{n}$. Then

$$
\begin{gathered}
\|u+\mathcal{V}\|_{C} \leq\|u\|_{C}+\|\mathcal{V}\|_{C}, \\
\|\lambda u\|_{C}=\lambda \cdot\|u\|_{C} \\
\|<\xi, u>\| \leq\|\xi\| \cdot\|u\|_{C} .
\end{gathered}
$$

Proof. The proofs of (1.21) and (1.22) are obvious. To prove inequality (1.23) it is enough to use Cauchy-Schwarz inequality. Thus,

$$
\begin{aligned}
\|<\xi, u>\| & =\left\|\sum_{i=1}^{n} \xi_{i} U_{i}\right\| \leq \sum_{i=1}^{n} \xi_{i}\left\|U_{i}\right\| \\
& \leq\left(\sum_{i=1}^{n}\left(\xi_{i}\right)^{2}\right)^{\frac{1}{2}} \cdot\left(\sum_{i=1}^{n}\left\|U_{i}\right\|^{2}\right)^{\frac{1}{2}}=\|\xi\| \cdot\|u\|_{C} .
\end{aligned}
$$

\section{Positive Linear Set Valued Maps}

Definition 1. Let $X$ and $Y$ be normed spaces, $K \subset X$ be defined by (1.1), $F(\cdot)$ : $K \rightarrow P_{k c}(Y)$ be a given set valued map. If

$$
F\left(\lambda x_{1}+\mu x_{2}\right)=\lambda F\left(x_{1}\right)+\mu F\left(x_{2}\right)
$$

for every $x_{1} \in K, x_{2} \in K, \lambda \in \mathbb{R}_{+}^{1}$ and $\mu \in \mathbb{R}_{+}^{1}$, then $F(\cdot)$ is called a positive linear set valued map.

Let us give the representation theorem for positive linear set valued maps.

Theorem 1. Let $K \subset X$ be defined by (1.1), $F(\cdot): K \rightarrow P_{k c}(Y)$ be a given positive linear set valued map. Then there exists a unique $\mathscr{F}=\left(F_{1}, F_{2}, \ldots, F_{n}\right) \in\left(P_{k c}(Y)\right)^{n}$ such that

$$
F(x)=<\mathbf{x}_{n}, \mathcal{F}>
$$

and

$$
\|F(x)\| \leq\left\|\mathbf{x}_{n}\right\| \cdot\|\mathcal{F}\|_{C} \leq \frac{1}{\beta}\|\mathcal{F}\|_{C} \cdot\|x\|_{X}
$$

for every $x \in K$, where $\beta>0$ is defined by (1.6). 
Proof. Denote $F_{i}=F\left(a_{i}\right)(i=1,2, \ldots, n)$ and $\mathcal{F}=\left(F_{1}, F_{2}, \ldots, F_{n}\right)$. Since $F_{i} \in$ $P_{k c}(Y)$, then $\mathscr{F} \in\left(P_{k c}(Y)\right)^{n}$.

Let $x \in K$. Then there exists a unique $\left(\xi_{1}, \xi_{2}, \ldots, \xi_{n}\right) \in \mathbb{R}_{+}^{n}$ such that $x=\sum_{i=1}^{n} \xi_{i} a_{i}$, and hence $\mathbf{x}_{n}=\left(\xi_{1}, \xi_{2}, \ldots, \xi_{n}\right)$. Since the set valued map $F(\cdot)$ is positive linear, we obtain

$$
F(x)=F\left(\sum_{i=1}^{n} \xi_{i} a_{i}\right)=\sum_{i=1}^{n} F\left(\xi_{i} a_{i}\right)=\sum_{i=1}^{n} \xi_{i} F\left(a_{i}\right)=\sum_{i=1}^{n} \xi_{i} F_{i}=<\mathbf{x}_{n}, \mathcal{F}>.
$$

The validity of inequality (2.2) follows from Proposition 1 and Proposition 3.

Now let us prove the uniqueness of representation (2.1). Let there exists $\mathbb{E}=$ $\left(E_{1}, E_{2}, \ldots, E_{n}\right) \in\left(P_{k c}(Y)\right)^{n}$ such that for every $x \in K$

$$
F(x)=\left\langle\mathbf{x}_{n}, \mathcal{E}>\right.\text {. }
$$

From (2.1) and (2.3) it follows that

$$
<\mathbf{x}_{n}, \mathcal{F}>=\left\langle\mathbf{x}_{n}, \mathcal{E}>\right.
$$

for every $x \in K$ and consequently

$$
\sum_{i=1}^{n} \xi_{i} F_{i}=\sum_{i=1}^{n} \xi_{i} E_{i}
$$

for every $\left(\xi_{1}, \xi_{2}, \ldots, \xi_{n}\right) \in \mathbb{R}_{+}^{n}$.

Taking $\xi_{i_{*}}=1, \xi_{i}=0$ if $i \neq i_{*}$, we get from (2.4) that $F_{i_{*}}=E_{i_{*}}$, and hence $F_{i}=$ $E_{i}$ for every $i=1,2, \ldots, n$. Thus $\mathcal{E}=\mathscr{F}$, and representation (2.1) is unique.

Theorem 1 implies the validity of the following corollary.

Corollary 1. Let $K \subset X$ be defined by $(1.1), F(\cdot): K \rightarrow P_{k c}\left(\mathbb{R}^{1}\right)$ be a given positive linear set valued map. Then there exists a unique $\mathcal{F}=\left(\left[\alpha_{1}, \beta_{1}\right],\left[\alpha_{2}, \beta_{2}\right], \ldots,\left[\alpha_{n}, \beta_{n}\right]\right) \in\left(P_{k c}\left(\mathbb{R}^{1}\right)\right)^{n}$ such that

$$
F(x)=\sum_{i=1}^{n} \xi_{i} \cdot\left[\alpha_{i}, \beta_{i}\right]=\left[\sum_{i=1}^{n} \xi_{i} \cdot \alpha_{i}, \sum_{i=1}^{n} \xi_{i} \cdot \beta_{i}\right]
$$

for every $x \in K$, where $\left(\xi_{1}, \xi_{2}, \ldots, \xi_{n}\right)=\mathbf{x}_{n}$ and $\left(\xi_{1}, \xi_{2}, \ldots, \xi_{n}\right) \in \mathbb{R}_{+}^{n}$.

\section{Lipschitz Continuity of the Positive Linear Set Valued Maps}

The following proposition characterizes Lipschitz continuity of the positive linear set valued maps. 
Theorem 2. Let $K \subset X$ be defined by $(1.1), F(\cdot): K \rightarrow P_{k c}(Y)$ be a given positive linear set valued map. Then the set valued map $F(\cdot)$ is Lipschitz continuous with Lipschitz constant $\frac{1}{\gamma}\|\mathcal{F}\|_{C}$ that is

$$
h_{Y}\left(F\left(x^{(1)}\right), F\left(x^{(2)}\right)\right) \leq \frac{1}{\gamma}\|\mathcal{F}\|_{C} \cdot\left\|x^{(1)}-x^{(2)}\right\|_{X}
$$

for every $x^{(1)} \in K, \quad x^{(2)} \in K$, where $\gamma>0$ is defined by (1.5), $\mathscr{F}=\left(F_{1}, F_{2}, \ldots, F_{n}\right) \in\left(P_{k c}(Y)\right)^{n}, F_{i} \in P_{k c}(Y)(i=1,2, \ldots, n)$ are defined in Theorem 1 .

Proof. According to Theorem 1, there exists a unique $\mathcal{F}=\left(F_{1}, F_{2}, \ldots, F_{n}\right) \in$ $\left(P_{k c}(Y)\right)^{n}$ such that for every $x \in K$

$$
F(x)=\left\langle\mathbf{x}_{n}, \mathcal{F}>.\right.
$$

Let $x^{(1)} \in K, \quad x^{(2)} \in K, \quad \mathbf{x}_{n}^{(1)}=\left(\xi_{1}^{(1)}, \xi_{2}^{(1)}, \ldots, \xi_{n}^{(1)}\right) \in \mathbb{R}_{+}^{n}$, $\mathbf{x}_{n}^{(2)}=\left(\xi_{1}^{(2)}, \xi_{2}^{(2)}, \ldots, \xi_{n}^{(2)}\right) \in \mathbb{R}_{+}^{n}$. Then we get from (3.1) that

$$
\begin{aligned}
& F\left(x^{(1)}\right)=<\mathbf{x}_{n}^{(1)}, \mathcal{F}>=\sum_{i=1}^{n} \xi_{i}^{(1)} F_{i}, \\
& F\left(x^{(2)}\right)=<\mathbf{x}_{n}^{(2)}, \mathcal{F}>=\sum_{i=1}^{n} \xi_{i}^{(2)} F_{i} .
\end{aligned}
$$

Let $u_{*} \in F\left(x^{(1)}\right)$ be an arbitrarily chosen vector. Then by virtue of (3.2) there exist $f_{1} \in F_{1}, f_{2} \in F_{2}, \ldots, f_{n} \in F_{n}$ such that

$$
u_{*}=\sum_{i=1}^{n} \xi_{i}^{(1)} f_{i} \text {. }
$$

Now let

$$
v_{*}=\sum_{i=1}^{n} \xi_{i}^{(2)} f_{i} .
$$

Then according to (3.3) we have $v_{*} \in F\left(x^{(2)}\right)$. From (3.4) and (3.5) it follows that

$$
\begin{aligned}
\left\|u_{*}-v_{*}\right\|_{Y}=\left\|\sum_{i=1}^{n}\left(\xi_{i}^{(1)} f_{i}-\xi_{i}^{(2)} f_{i}\right)\right\|_{Y} & \leq \sum_{i=1}^{n}\left|\xi_{i}^{(1)}-\xi_{i}^{(2)}\right|\left\|f_{i}\right\|_{Y} \\
& \leq \sum_{i=1}^{n}\left|\xi_{i}^{(1)}-\xi_{i}^{(2)}\right|\left\|F_{i}\right\| .
\end{aligned}
$$


(3.6) and Cauchy-Schwarz inequality yield

$$
\left\|u_{*}-v_{*}\right\|_{Y} \leq\left(\sum_{i=1}^{n}\left(\xi_{i}^{(1)}-\xi_{i}^{(2)}\right)^{2}\right)^{\frac{1}{2}} \cdot\left(\sum_{i=1}^{n}\left\|F_{i}\right\|^{2}\right)^{\frac{1}{2}}=\|\widetilde{F}\|_{C} \cdot\left\|\mathbf{x}_{n}^{(1)}-\mathbf{x}_{n}^{(2)}\right\|
$$

Since $x^{(1)}-x^{(2)} \in E^{n}$, where $E^{n}$ is defined by (1.2), Proposition 1 implies that

$$
\left\|\mathbf{x}_{n}^{(1)}-\mathbf{x}_{n}^{(2)}\right\| \leq \frac{1}{\gamma}\left\|x^{(1)}-x^{(2)}\right\|_{X},
$$

where $\gamma>0$ is defined by (1.5). (3.7) and (3.8) yield that

$$
\left\|u_{*}-v_{*}\right\|_{Y} \leq \frac{1}{\gamma}\|\widetilde{F}\|_{C} \cdot\left\|x^{(1)}-x^{(2)}\right\|_{X} .
$$

It follows from (3.9) that

$$
d_{Y}\left(u_{*}, F\left(x^{(2)}\right)\right) \leq \frac{1}{\gamma}\|\mathcal{F}\|_{C} \cdot\left\|x^{(1)}-x^{(2)}\right\|_{X} .
$$

Since $u_{*} \in F\left(x^{(1)}\right)$ is an arbitrarily chosen vector, then we get from (3.10) that

$$
\sup \left\{d_{Y}\left(u, F\left(x^{(2)}\right)\right): u \in F\left(x^{(1)}\right)\right\} \leq \frac{1}{\gamma}\|\mathcal{F}\|_{C} \cdot\left\|x^{(1)}-x^{(2)}\right\|_{X} .
$$

Analogously it is possible to prove that

$$
\sup \left\{d_{Y}\left(v, F\left(x^{(1)}\right)\right): v \in F\left(x^{(2)}\right)\right\} \leq \frac{1}{\gamma}\|\mathcal{F}\|_{C} \cdot\left\|x^{(1)}-x^{(2)}\right\|_{X} .
$$

(3.11) and (3.12) imply the proof of the proposition.

Proposition 4. Let $K \subset X$ be defined by (1.1), $F(\cdot): K \rightarrow P_{k c}(Y)$ be a given positive linear set valued map. Then

$$
\operatorname{gr} F(\cdot)=\{(x, y) \in K \times Y: y \in F(x)\}
$$

is a closed convex cone.

Proof. The proof that $g r F(\cdot)$ is a convex cone is obvious. Closedness of $g r F(\cdot)$ follows from Theorem 2, asserting that the set valued map $F(\cdot): K \rightarrow P_{k c}(Y)$ is continuous.

By virtue of [2], [11], and Proposition 4 we conclude that the positive linear set valued map $F(\cdot): K \rightarrow P_{k c}(Y)$ is a closed convex processes. 


\section{The Norm of the Positive Linear Set Valued Maps}

According to [2] and [6] the upper and lower norms of the positive linear set valued map $F(\cdot): K \rightarrow P_{k c}(Y)$, where $K \subset X$ is given by relation (1.1), are respectively defined as

$$
\begin{gathered}
\|F(\cdot)\|_{u}=\sup _{x \in K \backslash\left\{0_{X}\right\}} \frac{\|F(x)\|}{\|x\|_{X}}=\sup _{x \in K \backslash\left\{0_{X}\right\}} \frac{\sup \left\{\|f\|_{Y}: f \in F(x)\right\}}{\|x\|_{X}}, \\
\|F(\cdot)\|_{l}=\sup _{x \in K \backslash\left\{0_{X}\right\}} \frac{d_{Y}\left(0_{Y}, F(x)\right)}{\|x\|_{X}}=\sup _{x \in K \backslash\left\{0_{X}\right\}} \frac{\inf \left\{\|f\|_{Y}: f \in F(x)\right\}}{\|x\|_{X}} .
\end{gathered}
$$

It is obvious that $\|F(\cdot)\|_{l} \leq\|F(\cdot)\|_{u}$. The following theorem gives estimations of upper and lower norms of the positive linear set valued map $F(\cdot): K \rightarrow P_{k c}(Y)$.

Theorem 3. Let $K \subset X$ be defined by $(1.1), F(\cdot): K \rightarrow P_{k c}(Y)$ be a given positive linear set valued map. Then

$$
\max \left\{\frac{m_{i}}{\left\|a_{i}\right\|_{X}}: i=1,2, \ldots, n\right\} \leq\|F(\cdot)\|_{l} \leq\|F(\cdot)\|_{u} \leq \frac{1}{\beta}\|\mathscr{F}\|_{C},
$$

where $\beta>0$ is defined by (1.6), $\mathcal{F}=\left(F_{1}, F_{2}, \ldots, F_{n}\right) \in\left(P_{k c}(Y)\right)^{n}, F_{i} \in P_{k c}(Y)$ $(i=1,2, \ldots, n)$ are defined in Theorem $1, m_{i}=d_{Y}\left(0_{Y}, F_{i}\right), a_{i} \in X, i=1,2, \ldots, n$, are linearly independent vectors given in (1.1).

Proof. Applying Theorem 1 we have

$$
\begin{aligned}
\|F(\cdot)\|_{u} & =\sup _{x \in K \backslash\left\{0_{X}\right\}} \frac{\|F(x)\|}{\|x\|_{X}} \\
& \leq \sup _{x \in K \backslash\left\{0_{X}\right\}} \frac{1}{\beta}\|\mathcal{F}\|_{C}\|x\|_{X} \cdot \frac{1}{\|x\|_{X}}=\frac{1}{\beta}\|\mathcal{F}\|_{C} .
\end{aligned}
$$

Let us choose an $x \in K \backslash\left\{0_{X}\right\}$. Then there exists a unique $\left(\xi_{1}, \xi_{2}, \ldots, \xi_{n}\right) \in \mathbb{R}_{+}^{n}$ such that $x=\sum_{i=1}^{n} \xi_{i} a_{i}$, and hence $\mathbf{x}_{n}=\left(\xi_{1}, \xi_{2}, \ldots, \xi_{n}\right)$ and $\left\|\mathbf{x}_{n}\right\|=\left(\sum_{i=1}^{n} \xi_{i}^{2}\right)^{\frac{1}{2}}$.

Using Theorem 1 we have

$$
\begin{aligned}
d_{Y}\left(0_{Y}, F(x)\right) & =\min \left\{\|f\|_{Y}: f \in F(x)\right\}=\min \left\{\|f\|_{Y}: f \in<\mathbf{x}_{n}, \mathcal{F}>\right\} \\
& =\min \left\{\left\|\sum_{i=1}^{n} \xi_{i} f_{i}\right\|_{Y}: f_{i} \in F_{i}\right\}
\end{aligned}
$$


where $\mathcal{F}=\left(F_{1}, F_{2}, \ldots, F_{n}\right) \in\left(P_{k c}(Y)\right)^{n}, F_{i} \in P_{k c}(Y)(i=1,2, \ldots, n)$ are defined in Theorem 1. Since $x=\sum_{i=1}^{n} \xi_{i} a_{i}$, we obtain from (4.2) that

$$
\begin{aligned}
\frac{d_{Y}\left(0_{Y}, F(x)\right)}{\|x\|_{X}} & =\frac{\min \left\{\left\|\sum_{i=1}^{n} \xi_{i} f_{i}\right\|_{Y}: f_{i} \in F_{i}\right\}}{\left\|\sum_{i=1}^{n} \xi_{i} a_{i}\right\|_{X}} \\
& =\frac{\min \left\{\left\|\sum_{i=1}^{n} \frac{\xi_{i}}{\left\|\mathbf{x}_{n}\right\|} f_{i}\right\|_{Y}: f_{i} \in F_{i}\right\}}{\left\|\sum_{i=1}^{n} \frac{\xi_{i}}{\left\|\mathbf{x}_{n}\right\|} a_{i}\right\|_{X}} .
\end{aligned}
$$

Since $x \in K \backslash\left\{0_{X}\right\}$ is arbitrarily chosen, $\left(\frac{\xi_{1}}{\left\|\mathbf{x}_{n}\right\|}, \frac{\xi_{2}}{\left\|\mathbf{x}_{n}\right\|}, \ldots, \frac{\xi_{n}}{\left\|\mathbf{x}_{n}\right\|}\right) \in S_{+}^{n}$, (4.3) implies that

$$
\begin{aligned}
\|F(\cdot)\|_{l} & =\sup _{x \in K \backslash\left\{0_{X}\right\}} \frac{d_{Y}\left(0_{Y}, F(x)\right)}{\|x\|_{X}} \\
& =\sup _{x \in K \backslash\left\{0_{X}\right\}} \frac{\min \left\{\left\|\sum_{i=1}^{n} \frac{\xi_{i}}{\left\|\mathbf{x}_{n}\right\|} f_{i}\right\|_{Y}: f_{i} \in F_{i}\right\}}{\left\|\sum_{i=1}^{n} \frac{\xi_{i}}{\left\|\mathbf{x}_{n}\right\|} a_{i}\right\|_{X}} \\
& =\sup _{\left(\theta_{1}, \theta_{2}, \ldots, \theta_{n}\right) \in S_{+}^{n}} \frac{\min \left\{\left\|\sum_{i=1}^{n} \theta_{i} f_{i}\right\|_{Y}: f_{i} \in F_{i}\right\}}{\left\|\sum_{i=1}^{n} \theta_{i} a_{i}\right\|_{X}} .
\end{aligned}
$$

Let $\left(\theta_{1}^{*}, \theta_{2}^{*}, \ldots, \theta_{n}^{*}\right)=(0, \ldots, 0,1,0, \ldots, 0) \in S_{+}^{n}$ where 1 is $i$-th coordinate. Then it follows from (4.4) that

$$
\|F(\cdot)\|_{l}=\sup _{\left(\theta_{1}, \theta_{2}, \ldots, \theta_{n}\right) \in S_{+}^{n}} \frac{\min \left\{\left\|\sum_{i=1}^{n} \theta_{i} f_{i}\right\|_{Y}: f_{i} \in F_{i}\right\}}{\left\|\sum_{i=1}^{n} \theta_{i} a_{i}\right\|_{X}}
$$




$$
\begin{gathered}
\geq \frac{\min \left\{\left\|\sum_{i=1}^{n} \theta_{i}^{*} f_{i}\right\|_{Y}: f_{i} \in F_{i}\right\}}{\left\|\sum_{i=1}^{n} \theta_{i}^{*} a_{i}\right\|_{X}} \\
=\frac{\min \left\{\left\|f_{i}\right\|_{Y}: f_{i} \in F_{i}\right\}}{\left\|a_{i}\right\|_{X}}=\frac{m_{i}}{\left\|a_{i}\right\|_{X}} .
\end{gathered}
$$

Since the inequality (4.5) holds for every $i=1,2, \ldots, n$, then we have from (4.5) that

$$
\|F(\cdot)\|_{l} \geq \max \left\{\frac{m_{i}}{\left\|a_{i}\right\|_{X}}: i=1,2, \ldots, n\right\} .
$$

The inequalities (4.1) and (4.6) complete the proof.

\section{ACKNOWLEDGMENT}

We would like to thank the anonymous reviewers for their valuable comments and suggestions.

\section{REFERENCES}

[1] J.-P. Aubin, H. Frankowska, and C. Olech, "Controllability of convex processes," SIAM J. Control Optimization, vol. 24, pp. 1192-1211, 1986.

[2] J.-P. Aubin and H. Frankowska, Set-valued analysis, ser. Systems and Control: Foundations and Applications. Boston: Birkhäuser, 1990, vol. 2.

[3] J. M. Borwein and A. S. Lewis, Convex analysis and nonlinear optimization. Theory and examples, ser. CMS Books in Mathematics/Ouvrages de Mathématiques de la SMC. New York: Springer, 2000, vol. 3 .

[4] E. Ekici, "On the directional derivative and directional continuity of set valued maps," Miskolc Math. Notes, vol. 6, no. 2, pp. 173-183, 2005.

[5] A. D. Ioffe, "Nonsmooth analysis and the theory of fans. Convex analysis and optimization," Res. Notes in Math., vol. 57, pp. 93-117, 1982.

[6] A. D. Ioffe, "On regularity of convex multifunctions," Nonlinear Anal., Theory Methods Appl., vol. 69, no. 3, pp. A, 843-849, 2008.

[7] M. D. Monteiro Marques, Differential inclusions in nonsmooth mechanical problems. Shocks and dry friction, ser. Progress in Nonlinear Differential Equations and their Applications. Basel: Birkhäuser, 1993, vol. 9.

[8] S. M. Robinson, "Normed convex processes," Trans. Am. Math. Soc., vol. 174, pp. 127-140, 1972.

[9] S. M. Robinson, "Regularity and stability for convex multivalued functions," Math. Oper. Res., vol. 1, pp. 130-143, 1976.

[10] R. T. Rockafellar, "Monotone processes of convex and concave type," Mem. Am. Math. Soc., vol. 77, p. 74, 1967.

[11] R. T. Rockafellar, Convex analysis. Princeton, N. J.: Princeton University Press, 1970.

[12] C. Ursescu, "Multifunctions with convex closed graph," Czech. Math. J., vol. 25(100), pp. 438 $441,1975$. 
Authors' addresses

Anar Huseyin

Anadolu University, Mathematics Department, 26490 Eskisehir, Turkey

E-mail address: ahuseyineanadolu.edu.tr

Nesir Huseyin

Anadolu University, Mathematics Department, 26490 Eskisehir, Turkey

E-mail address: nhuseyin@anadolu.edu.tr 